\title{
Towards An Accessible Life Cycle Assessment: A Literature Based Review Of Current BIM And Parametric Based Tools Capabilities
}

\author{
Carmine Cavalliere ${ }^{1}$, Leonardo Brescia ${ }^{1,2}$, Gaetano Maiorano ${ }^{1,2}$, Tiziano Dalla Mora $^{3}$, Guido \\ Raffaele Dell'Osso ${ }^{1}$, Emanuele Naboni ${ }^{2}$ \\ ${ }^{1}$ Polytechnic University of Bari, Bari, Italy \\ ${ }^{2}$ The Royal Danish Academy of Fine Arts Schools of Architecture, Design and Conservation, \\ Copenhagen, Denmark \\ ${ }^{3}$ University IUAV of Venice, Venice, Italy
}

\begin{abstract}
Life Cycle Assessment (LCA) for buildings has not been widely applied because of its complexity and timeconsuming nature. Several studies have been trying to address this issue by the use of Building Information Modelling (BIM) and Parametric tools, which enable them to face specific issues arising on projects. Amongst the different research efforts, one can perceive a gap in the field. This gap lies in the insufficient understanding of the methodological approaches and tools best suited to carry out environmental analysis. The novelty of the paper is to compare the paradigms and scope of most common used BIM and Parametric approach for LCA. This leads to an enhanced awareness on how to apply both trending phenomena to increase their features and reduce the limitations.
\end{abstract}

\section{Introduction}

The Architecture, Engineering and Construction (AEC) sector is one of the major carbon emitters and energy consumers. Several tools and methodologies have been developed to evaluate the environmental impact of buildings and there is a growing interest in integrating Life Cycle Assessment (LCA) into building design decision-making. LCA evaluates the environmental impact of processes and products, by means of some indicators that evaluate the consumption of raw materials, energy and emissions in the environment during their life cycle, from raw materials extraction and processing to the use and end of life stage. However, several challenges and limitations for building's LCA could be found along their design process.

Building Information Modelling (BIM) is seen as an approach that can assist the building community in accomplishing the sustainability objectives. Existing studies show the possibility of conducting a BIM-based LCA and by that overcoming some of the limitations (Cavalliere et al., 2018; Chong et al., 2017; Eleftheriadis et al., 2017; Kylili et al., 2015; Soares et al., 2017; SoustVerdaguer et al., 2017; Wong and Zhou, 2015). For example, BIM can reduce the time-consuming nature of the LCA for collecting data as it allows for performing quick quantity take-off (Ajayi et al., 2015; Houlihan Wynberg et al., 2014). Next to the BIM-based approach, Parametric approaches such as these based in Rhino/Grasshopper and Dynamo are considered of high potential for performing LCA. These are visual Programming Language software tools that have the potential to include geometric modelling and scripting functionalities useful when more environmental impact categories are needed. Furthermore, optimization processes could be performed. (Hollberg and Ruth, 2016).

BIM and Parametric-based approaches for LCA are significantly different due to the type of tools employed and their functionalities. The goal of this paper is to provide a framework to evaluate their differences on the basis of the latest published papers in the field. This helps to understand which methodological approach and tool best suited to perform the environmental analysis, concerning the practitioner's knowledge and data availability.

\section{Life Cycle Assessment}

The Life Cycle Assessment is today a very useful environmental assessment method as an instrument accepted by the international scientific community and recognized as suitable for identifying the strategies necessary for companies to become environmentally efficient in terms of reducing resource and energy consumption and minimize the effects on the environment. From this derives the choice, by the research network, to define guidelines for the environmental assessment of buildings based on this methodology.

\section{BIM and Parametric tools}

Building Information Modelling (BIM) is the primary vector whereby the AEC sector is moving towards the digital prototyping. BIM is oriented to the modelling and sharing of both graphical and non-graphical data, allowing the extraction of quantities, material properties and cost estimations for building, facility and infrastructures (Cheung et al., 2012). Hence, BIM has the potential to manage project alternatives bringing forward the design choices through the early performance design analysis (Schade et al., 2011). BIM is a radically different system for collecting, using and sharing data on the life cycle of a building following a repeatable and verifiable decision-making process, which reduces risks and improves the quality of products on an industrial level.

Next to the spread of BIM, a new tools evolution is taking place in the field of AEC. Visual Programming Language (VPL) tools allow programming through a graphical manipulation of elements, rather than written syntax. Architects and engineers are increasingly using VPL as scripting since it defines the parametric method of design exploration and optimization. Hence, 
Parametric tools are powerful tools to increase the productivity of building designers due to their ability to automate the generation of geometric compositions (Negendahl, 2015). Current developments in this area aim to integrate the visual programming approach with dataflow programming languages in order to automate the documentation.

\section{Method}

This paper examines recent studies focusing on the BIM/Parametric approach to conduct LCA for buildings in order to highlight the weakness and strengths of both methods. the paper analyses the research on BIM and Parametric-based LCA (PLCA) that have been published from 2013 to 2018 on the main scientific article collection platforms (ScienceDirect, Scopus, ResearchGate, etc). The comparison between BIM and Parametric-based approach for LCA is based on different criteria, which are defined in the following.

\section{Analysis criteria}

This paper proposes four different criteria for the evaluation of the model. These were selected on the basis of the information extracted from the analyzed documents and concern the physical characteristics of the building model taken up in the processes and phases by the LCA method. The first and second are the approaches and the design stages considered in the reviewed paper highlighted to identify which approach best suited the different design phases. The definition of the Level of Development (LOD) is the third criterion considered. LOD defines the minimum information content for each element of the BIM (or VPL as appropriate) at the different progressively detailed level of completeness. According to Soust-Verdaguer et al. (2017), LODs are of high importance when conducting a BIM-based LCA since it indicates the LCA data requirements of the model. The last one is the used tool considered as a crucial aspect when analyzing the different approaches. For example, the type and the number of tools represent the complexity of the approach adopted. Four criteria are also considered on the LCA side. The functional unit (FU) is taken as a criterion to compare the reviewed paper. The FU is a quantified performance of a system used as a reference unit (ISO 14040, 2006). In this regard, for each case analyzed, the FU is reported to point out if simplifications are made according to different approaches. On the same grounds, other criteria have been analyzed here. They are the environmental impacts and the LCA phases considered. Finally, the database employed is considered as an additional indicator showing the complexity of the approaches and the level of interoperability reached.

\section{Results}

The main findings of the research are summarized in Table 1 and they are discussed in the following.

\section{Approach adopted (BIM or Parametric)}

The literature review shows the increasing interest in performing LCA based on BIM and Parametric approach. In particular, the Parametric approach is detected as a relatively new approach to perform LCA of buildings with most papers published in 2018. However, as can be seen from Table 1, most of the papers are still based on building information modelling to perform lifecycle analysis and the trend is continuously growing.

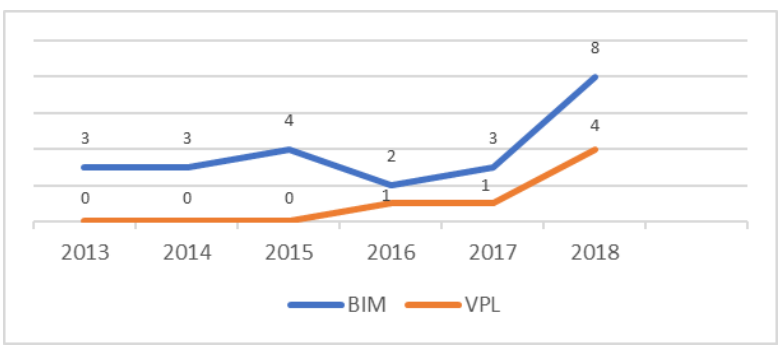

Figure 1: Trends in BIM/VPL-based papers.

\section{Design stages}

According to Cavalliere et al. (2019), two different trends exist to perform LCA of buildings based on the digital model. The first trend concerns on performing detailed LCA with refined processes and specific building simulation tools. On the contrary, the second trend involves simplified approaches for early design stages. Existing literature for both trends is reviewed and summarized in Table 1. Although new methods for performing LCA over the entire design stage exists, they are seldom discussed. Cavalliere et al. (2019) proposes a novel method for applying LCA continuously over the design process using the data provided by BIM with the optimal accuracy that is appropriate to each design stage. The method uses different LCA databases with different Levels of Development (LODs) of the BIM, which is possible as long as the databases use identical background data. The Parametric-based papers analyzed to focus on the early design stage, while the BIM- based approach refers to both trends depending on the tools used. Only Shadram and Mukkavaara (2018) uses a VPL-based coupled with BIM tools for performing LCA in the detailed stage.

\section{Level of Development (LOD)}

As seen in Table 1, only few studies set a fixed LOD. Ajayi et al. (2015) and Röck et al. (2018) were based on a LOD 200 model to support early environmental analysis. LOD 300 was declared in two cases to support detailed analysis (Lee et al., 2015; Yang et al., 2018). Amongst the papers with Parametric-based approach, only Röck et al. (2018) defines the LOD of the model elements. It can be observed that the paper only defines a single level of development since they refer to a single design stage. Cavalliere et al. (2019) uses four LOD (from 100 to 400) as the study refers to a framework for conducting a BIM-based LCA during the entire design process.

\section{Tools used}

Despite the geographical heterogeneity of the case studies, most of the reviewed papers used Autodesk Revit software to develop the model, today the most widespread in the world, which fully expresses the potential of the BIM approach in engineering and architecture. 
Table 1: Summary of the reviewed paper.

\begin{tabular}{|c|c|c|c|c|c|c|c|c|c|c|}
\hline \multirow{2}{*}{ Reference } & \multicolumn{2}{|c|}{ Approaches } & \multicolumn{2}{|c|}{ Design stages } & \multirow[t]{2}{*}{ LOD } & \multirow[t]{2}{*}{ Tools } & \multirow[t]{2}{*}{ Impact } & \multirow[t]{2}{*}{ FU } & \multirow[t]{2}{*}{ DB } & \multirow[t]{2}{*}{$\begin{array}{l}\text { LCA } \\
\text { phase }\end{array}$} \\
\hline & BIM & Par. & Early & Detailed & & & & & & \\
\hline $\begin{array}{l}\text { (Abanda } \\
\text { et al., 2017) }\end{array}$ & $\mathrm{x}$ & & & $\mathrm{x}$ & - & $\begin{array}{c}\text { Revit, } \\
\text { Navisworks, } \\
\text { Excel, } \\
\text { API }\end{array}$ & $\begin{array}{l}\text { ECOE; } \\
\text { EE }\end{array}$ & $\begin{array}{l}\text { Complete } \\
\text { building }\end{array}$ & ICE & - \\
\hline $\begin{array}{c}\text { (Ajayi } \\
\text { et al., 2015) }\end{array}$ & $\mathrm{x}$ & & $\mathrm{x}$ & & 200 & $\begin{array}{c}\text { Revit, } \\
\text { Green Building } \\
\text { Studio, } \\
\text { ATHENA } \\
\text { Impact } \\
\text { Estimator, } \\
\text { Excel } \\
\end{array}$ & $\begin{array}{l}\text { GWP; } \\
\text { HH }\end{array}$ & $\begin{array}{l}\text { Complete } \\
\text { building }\end{array}$ & $\begin{array}{l}\text { ATHENA } \\
\text { Impact } \\
\text { Estimator }\end{array}$ & $\begin{array}{l}\text { A1-A3, } \\
\text { A4-A5, } \\
\text { B1-B7, } \\
\text { C1-C4 }\end{array}$ \\
\hline $\begin{array}{l}\text { (Basbagill } \\
\text { et al., 2017) }\end{array}$ & $\mathrm{x}$ & & $\mathrm{x}$ & & - & $\begin{array}{c}\text { Dprofiler, } \\
\text { CostLab, } \\
\text { eQUEST, } \\
\text { SimaPro, } \\
\text { ATHENA } \\
\text { EcoCalculator } \\
\text { Excel } \\
\end{array}$ & EIF & $\begin{array}{l}\text { Complete } \\
\text { building }\end{array}$ & $\begin{array}{l}\text { Athena Eco } \\
\text { Calculator }\end{array}$ & $\begin{array}{l}\text { A1-A3, } \\
\text { B1-B7 }\end{array}$ \\
\hline $\begin{array}{l}\text { (Bueno } \\
\text { et al., 2018) }\end{array}$ & $\mathrm{x}$ & $\mathrm{x}$ & $\mathrm{x}$ & & - & $\begin{array}{c}\text { Revit, } \\
\text { Dynamo, } \\
\text { Excel }\end{array}$ & $\begin{array}{c}\text { ReCiPe } \\
\text { midpoint } \\
\text { indicators }\end{array}$ & $\begin{array}{l}\text { Walls and } \\
\text { roofing } \\
\text { systems }\end{array}$ & Ecoinvent & - \\
\hline $\begin{array}{c}\text { (Cavalliere et } \\
\text { al., 2019) }\end{array}$ & $\mathrm{x}$ & & $\mathrm{x}$ & $\mathrm{x}$ & $\begin{array}{l}100 \text { to } \\
400\end{array}$ & $\begin{array}{l}\text { 3D model, } \\
\text { Excel }\end{array}$ & GWP & $\begin{array}{l}\text { Complete } \\
\text { building }\end{array}$ & $\begin{array}{c}\text { Swiss } \\
\text { building db, } \\
\text { KBOB, } \\
\text { Bauteilkat }\end{array}$ & $\begin{array}{l}\text { A1-A3, } \\
\text { B4, } \\
\text { C3, } \\
\text { C4 }\end{array}$ \\
\hline $\begin{array}{l}\text { (Eleftheriadis } \\
\text { et al., 2018) }\end{array}$ & $\mathrm{x}$ & & $\mathrm{x}$ & & - & $\begin{array}{l}\text { Revit, } \\
\text { Excel }\end{array}$ & ECE & $1 \mathrm{~m}^{2}$ of GFA & EPD & A1-A3 \\
\hline $\begin{array}{l}\text { (Georges } \\
\text { et al., 2015) }\end{array}$ & $\mathrm{x}$ & & & $\mathrm{x}$ & - & $\begin{array}{c}\text { Revit, } \\
\text { Excel, } \\
\text { SIMIEN, } \\
\text { SimaPro } 7.3 \\
\end{array}$ & $\begin{array}{l}\text { ECOE; } \\
\text { OCOE }\end{array}$ & $1 \mathrm{~m}^{2}$ of $\mathrm{HFA}$ & \begin{tabular}{c|} 
Ecoinvent \\
Version 2.2
\end{tabular} & $\begin{array}{l}\text { A1-A3, } \\
\text { B1, } \\
\text { B4, } \\
\text { B6 }\end{array}$ \\
\hline $\begin{array}{c}\text { (Hollberg and } \\
\text { Ruth, 2016) }\end{array}$ & & $\mathrm{x}$ & $\mathrm{x}$ & & - & $\begin{array}{l}\text { Grasshopper, } \\
\text { Rhinoceros }\end{array}$ & $\begin{array}{c}\text { PET; } \\
\text { PERT; } \\
\text { PENRT; } \\
\text { GWP; } \\
\text { EP; } \\
\text { AP; } \\
\text { ODP; } \\
\text { POCP; } \\
\text { ADPE } \\
\end{array}$ & $\begin{array}{l}\text { Complete } \\
\text { building }\end{array}$ & $\begin{array}{l}\text { ökobau.dat, } \\
\text { EPDs }\end{array}$ & $\begin{array}{l}\text { A1-A3, } \\
\text { B4, } \\
\text { B6, } \\
\text { C3, } \\
\text { C4 }\end{array}$ \\
\hline $\begin{array}{l}\text { (Houlihan } \\
\text { Wiberg } \\
\text { et al., 2014) }\end{array}$ & $\mathrm{x}$ & & & $\mathrm{x}$ & - & $\begin{array}{c}\text { Revit, } \\
\text { Excel, } \\
\text { SIMIEN, } \\
\text { SimaPro } 7.3 \\
\end{array}$ & $\begin{array}{l}\text { ECOE; } \\
\text { OCOE }\end{array}$ & $1 \mathrm{~m}^{2}$ of $\mathrm{HFA}$ & $\begin{array}{c}\text { Ecoinvent } \\
\text { Version } 2.2\end{array}$ & $\begin{array}{l}\text { A1-A3, } \\
\text { B4, } \\
\text { B6 }\end{array}$ \\
\hline $\begin{array}{c}\text { (Iddon and } \\
\text { Firth, 2013) }\end{array}$ & $\mathrm{x}$ & & & $\mathrm{x}$ & - & $\begin{array}{c}\text { BIM tool }(\mathrm{N} / \mathrm{S}) \\
\text { Excel }\end{array}$ & $\begin{array}{l}\text { ECOE; } \\
\text { OCOE }\end{array}$ & $\begin{array}{l}\text { Complete } \\
\text { building }\end{array}$ & ICE & $\begin{array}{l}\text { A1-A3, } \\
\text { B6 }\end{array}$ \\
\hline $\begin{array}{l}\text { (Jalaei and } \\
\text { Jrade, 2014) }\end{array}$ & $\mathrm{x}$ & & $\mathrm{x}$ & & - & $\begin{array}{c}\text { Revit, } \\
\text { Ecotect, } \\
\text { IESVE, } \\
\text { Excel, } \\
\text { Athena Impact } \\
\text { Estimator }\end{array}$ & $\begin{array}{l}\text { AP; } \\
\text { EP; } \\
\text { GWP; } \\
\text { HH; } \\
\text { ODP; } \\
\text { PEC; }\end{array}$ & $\begin{array}{l}\text { Complete } \\
\text { building }\end{array}$ & $\begin{array}{l}\text { ATHENA } \\
\text { Impact } \\
\text { Estimator }\end{array}$ & $\begin{array}{l}\text { A1-A3, } \\
\text { B6 }\end{array}$ \\
\hline
\end{tabular}




\begin{tabular}{|c|c|c|c|c|c|c|c|c|c|c|}
\hline $\begin{array}{c}\text { (Jrade and } \\
\text { Jalaei, 2013) }\end{array}$ & $\mathrm{x}$ & & $\mathrm{x}$ & & - & \begin{tabular}{|c|} 
Revit, \\
Athena Impact \\
Estimator, Excel
\end{tabular} & $\begin{array}{c}\text { PCSP; } \\
\text { REP; } \\
\text { WRRU } \\
\end{array}$ & $\begin{array}{l}\text { Complete } \\
\text { building }\end{array}$ & & $\begin{array}{l}\text { A1-A3, } \\
\text { B1-B7 }\end{array}$ \\
\hline $\begin{array}{c}\text { (Lee } \\
\text { et al., 2015) }\end{array}$ & $\mathrm{x}$ & & & $\mathrm{x}$ & 300 & $\begin{array}{l}\text { Revit, } \\
\text { Korea LCI } \\
\text { database }\end{array}$ & $\begin{array}{l}\text { ADP; } \\
\text { AP; } \\
\text { EP; } \\
\text { GWP; } \\
\text { ODP; } \\
\text { POCP }\end{array}$ & $\begin{array}{l}\text { Complete } \\
\text { building }\end{array}$ & Korean LCI & $\begin{array}{l}\text { A1-A3, } \\
\text { A4-A5, } \\
\text { B1-B7, } \\
\text { C1-C4 }\end{array}$ \\
\hline $\begin{array}{l}\text { (Lobaccaro } \\
\text { et al., 2018) }\end{array}$ & & $\mathrm{x}$ & $\mathrm{x}$ & & - & \begin{tabular}{|c|} 
Grasshopper, \\
Evaluate, \\
Design Builder, \\
DIVA, Ladybug, \\
Galapagos, \\
Octopus, \\
Rhinoceros \\
\end{tabular} & GWP & $1 \mathrm{~m}^{2}$ of HFA & $\begin{array}{c}\text { EPD } \\
\text { Norway, } \\
\text { Ecoinvent }\end{array}$ & $\begin{array}{l}\text { A1-A5, } \\
\text { B4, } \\
\text { B6 }\end{array}$ \\
\hline $\begin{array}{l}\text { (Marzouk } \\
\text { et al., 2017) }\end{array}$ & $\mathrm{x}$ & & & $\mathrm{x}$ & - & \begin{tabular}{|c|} 
Revit, \\
Revit DB link, \\
MS Access, \\
Athena Impact \\
Estimator, \\
MS Excel, \\
Visual Studio \\
\end{tabular} & $\begin{array}{l}\mathrm{CO} 2 \\
\text { SO2; } \\
\text { PM; } \\
\text { EP; } \\
\text { ODP; } \\
\text { PSP }\end{array}$ & $\begin{array}{l}\text { Complete } \\
\text { building }\end{array}$ & $\begin{array}{l}\text { ATHENA } \\
\text { Impact } \\
\text { Estimator }\end{array}$ & $\begin{array}{l}\text { A1-A3, } \\
\text { A4-A5, } \\
\text { B1-B7, } \\
\text { C1-C4 }\end{array}$ \\
\hline $\begin{array}{l}\text { (Najjar } \\
\text { et al., 2017) }\end{array}$ & $\mathrm{x}$ & & $\mathrm{x}$ & & - & $\begin{array}{c}\text { Revit, } \\
\text { Tally, } \\
\text { Green Building } \\
\text { Studio }\end{array}$ & $\begin{array}{c}\text { AP; } \\
\text { EP; } \\
\text { GWP; } \\
\text { ODP; } \\
\text { SMP; } \\
\text { PET; } \\
\text { PERT; } \\
\text { PENRT }\end{array}$ & $\begin{array}{l}\text { Complete } \\
\text { building }\end{array}$ & $\begin{array}{c}\text { GaBi } \\
\text { database }\end{array}$ & $\begin{array}{l}\text { A1-A3, } \\
\text { B1-B7, } \\
\text { C1-C4 }\end{array}$ \\
\hline $\begin{array}{l}\text { (Nizam } \\
\text { et al., 2018) }\end{array}$ & $\mathrm{x}$ & & $\mathrm{x}$ & & - & $\begin{array}{c}\text { Revit, } \\
\text { Revit API, } \\
\text { External } \\
\text { databases }\end{array}$ & $\mathrm{EE}$ & $\begin{array}{c}\text { Complete } \\
\text { building }\end{array}$ & $\begin{array}{c}\text { ICE, Chinese } \\
\text { handbook }\end{array}$ & $\begin{array}{l}\text { A1-A3, } \\
\text { A4-A5 }\end{array}$ \\
\hline $\begin{array}{l}\text { (Panteli } \\
\text { et al., 2018) }\end{array}$ & $\mathrm{x}$ & & $\mathrm{x}$ & & - & $\begin{array}{l}\text { Revit, } \\
\text { Insight, }\end{array}$ & $\begin{array}{c}\text { GWP; } \\
\text { AP; } \\
\text { EP; } \\
\text { ODP; } \\
\text { ADPele; } \\
\text { ADPfoss; } \\
\text { TETP } \\
\text { FAETP; } \\
\text { HTTP; } \\
\text { MAETP; } \\
\text { POCP; }\end{array}$ & $\begin{array}{l}\text { Complete } \\
\text { building }\end{array}$ & EcoHestia & $\begin{array}{l}\text { A1-A3, } \\
\text { A4-A5 }\end{array}$ \\
\hline (Peng, 2016) & $\mathrm{x}$ & & & $\mathrm{x}$ & - & $\begin{array}{l}\text { Revit, } \\
\text { Ecotect, } \\
\text { Excel }\end{array}$ & $\mathrm{COE}$ & $\begin{array}{l}\text { Complete } \\
\text { building }\end{array}$ & ICE & $\begin{array}{l}\text { A1-A3, } \\
\text { A4-A5, } \\
\text { B1-B7, } \\
\text { C1-C4 }\end{array}$ \\
\hline $\begin{array}{c}\text { (Röck } \\
\text { et al., 2018) }\end{array}$ & $\mathrm{x}$ & $\mathrm{x}$ & $\mathrm{x}$ & & 200 & $\begin{array}{c}\text { Revit, } \\
\text { Dynamo, } \\
\text { Excel }\end{array}$ & GWP & $1 \mathrm{~m}^{2}$ of GFA & Ecoinvent & $\begin{array}{c}\text { A1-A3, } \\
\text { B4, } \\
\text { C3-C4 }\end{array}$ \\
\hline $\begin{array}{l}\text { (Shadram } \\
\text { et al., 2016) }\end{array}$ & $\mathrm{x}$ & & & $\mathrm{x}$ & - & $\begin{array}{c}\text { Revit, } \\
\text { Power Pivot, } \\
\text { FME, Google } \\
\text { Maps API }\end{array}$ & $\mathrm{EE}$ & External wall & $\begin{array}{c}\text { EPD } \\
\text { database }\end{array}$ & $\begin{array}{c}\mathrm{A} 1-\mathrm{A} 3, \\
\mathrm{~A} 4\end{array}$ \\
\hline $\begin{array}{c}\text { (Shadram and } \\
\text { Mukkavaara, } \\
\text { 2018) }\end{array}$ & $\mathrm{x}$ & $\mathrm{x}$ & & $\mathrm{x}$ & - & $\begin{array}{c}\text { Revit, Dynamo, } \\
\text { MySQL, } \\
\text { Grasshopper, } \\
\text { Slingshot, } \\
\text { Archsim, } \\
\text { Octopus, } \\
\text { EnergyPlus }\end{array}$ & $\begin{array}{l}\mathrm{EE}, \\
\mathrm{OE}\end{array}$ & $\begin{array}{l}\text { Complete } \\
\text { building }\end{array}$ & ICE & $\begin{array}{l}\text { A1-A3, } \\
\text { B1, } \\
\text { B4, } \\
\text { B6, } \\
\text { B7 }\end{array}$ \\
\hline
\end{tabular}




\begin{tabular}{|c|c|c|c|c|c|c|c|c|c|}
\hline $\begin{array}{c}\text { (Shafiq } \\
\text { et al., 2015) }\end{array}$ & $\mathrm{x}$ & $\mathrm{x}$ & & - & $\begin{array}{l}\text { Revit, } \\
\text { Excel }\end{array}$ & $\mathrm{ECOE}$ & $\begin{array}{c}\text { Complete } \\
\text { building }\end{array}$ & ICE & $\begin{array}{c}\mathrm{A} 1-\mathrm{A} 3, \\
\mathrm{~A} 4\end{array}$ \\
\hline $\begin{array}{c}\text { (Shin and Cho, } \\
\text { 2015) }\end{array}$ & $\mathrm{x}$ & $\mathrm{x}$ & & - & $\begin{array}{c}\text { ArchiCAD, } \\
\text { Excel }\end{array}$ & $\mathrm{COE}$ & $\begin{array}{c}\text { Complete } \\
\text { building }\end{array}$ & Korean LCI & $\begin{array}{l}\text { A1-A3, } \\
\text { B1-B6 }\end{array}$ \\
\hline $\begin{array}{c}\text { (Yang } \\
\text { et al., 2018) }\end{array}$ & $\mathrm{x}$ & & $\mathrm{x}$ & 300 & $\begin{array}{c}\text { Revit, } \\
\text { Excel } \\
\text { Glondon } \\
\text { BIM5D, } \\
\text { eBALANCE, } \\
\text { Designbuilder, }\end{array}$ & GWP & $\begin{array}{c}\text { Complete } \\
\text { building }\end{array}$ & $\begin{array}{l}\text { Chinese db, } \\
\text { Ecoinvent, } \\
\text { ELCD }\end{array}$ & $\begin{array}{l}\text { A1-A3, } \\
\text { A4-A5, } \\
\text { B1-B7, } \\
\text { C1-C4 }\end{array}$ \\
\hline
\end{tabular}

Abbreviations: ADP, Abiotic Depletion Potential; ADPele, Abiotic Depletion Potential - elements; ADPfoss, Abiotic Depletion Potential - fossil; AP, Acidification Potential; Ap, Acidification process; COE, CO2 Emissions; ECE, Embodied Carbon Emission; ECOE, Embodied CO2 Emissions; EE, Embodied Energy; EFP, Effects Potential; EIF, Embodied Impact Factor; EP, Eutrophication Potential; Ep, Eutrophication process; FAETP, Fresh water Aquatic Ecotoxicity Potential; GHG, Greenhouse Gases; GWP, Global Warming Potential; HH, Human Health; HTP, Human toxicity potential; MAETP, Marine Aquatic Ecotoxicity Potential; OCOE, Embodied CO2 Emissions; ODp, Ozone Depleting particles; ODP, Ozone Depletion Potential; PET, Total Primary Energy; PCSP, Photo-chemical Smog Potential; PEC, Primary Energy Consumption; PERT, Total renewable primary energy; PENRT, Total NonRenewable Primary Energy; PM, Particular Matter; POCP, Photochemical Ozone Creation Potential; REP, Respiratory Effects Potential; Smog, Smog; SMP, Smog Formation Potential; WRRU, Weighted Raw Resource Use.

The use of different BIM software was detected in few studies. Basbagill et al. (2013) used the DProfiler as a BIM software while Shin and Cho (2015)

employed ArchiCAD 15. The most widely Parametric tools are Dynamo and Grasshopper, which can be directly linked with Revit and Rhinoceros respectively.

\section{Environmental impact considered}

ISO standards do not prescribe an impact category list. Nevertheless, they recommend that impact categories, category indicator sand characterization models have to be internationally accepted, based on international agreement or recognized by an authorized international board. The section of the impact categories depends on the goal and scope (G\&S) and the authors of the LCA. Table 1 shows the environmental indicators chosen by the reviewed papers. Regarding the reviewed papers, the most calculated environmental impact indicator was the Global Warming Potential (GWP) both in the case of BIM and Parametric approaches. Sometimes it is used as a single indicator (Cavalliere et al., 2019; Lobaccaro et al., 2018; Röck et al., 2018; Yang et al., 2018). Other BIM-based studies refer to a single environmental indicator considering Embodied Impact Factor (EIF) (Basbagill et al., 2013), Embodied Carbon Emission (ECE) (Eleftheriadis et al., 2018), Embodied Energy (Nizam et al., 2018; Shadram et al., 2016), CO2 Emission (COE) (Peng, 2016; Shin and Cho, 2015), and Embodied CO2 Emission (ECOE) (Shafiq et al., 2015).

\section{Functional Unit}

According to the EN 15978:2011 (EN 15978, 2011), the functional equivalent defines the required technical characteristics and functionalities of buildings or building components. A variety of functional units is used in LCA of buildings (Cabeza et al., 2014). Meter square and the whole building are reported as the most used FU in the case of BIM-based approach. Several studies consider the complete building as a FU, and in other cases, the FU is a part of the building, such as the walls and roofing systems (Bueno et al., 2018; Shadram et al., 2016). Meter square of Heated Floor Area (HFA) and meter square of Gross Floor Area (GFA) are also used as FU in the case of BIM approach. Life Cycle Assessment studies conducted by the aid of Parametric tools considered various FU as well. Hollberg and Ruth (2016) refer to the complete building for the assessment, while Lobaccaro et al. (2018) considered $1 \mathrm{~m} 2$ of HFA as a FU. Other studies setting a workflow based on both BIM and Parametric tools refer to the whole building (Shadram and Mukkavaara, 2018), part of the building (Bueno et al., 2018), and $1 \mathrm{~m} 2$ of GFA (Röck et al., 2018).

\section{Databases employed}

There are various multi-sectorial generic LCI databases, such as ELCD database, Ecoinvent database, GaBi database. Databases explicitly developed for the construction industry have also been published in the last years and are usually employed. Table 1 lists the most used LCA databanks for the construction sector, which are often included within LCA software tools.

\section{LCA phase}

EN 15978:2011 is structured according to the "life-cycle modules" of buildings, including four stages: Product (A1 -A3), Construction process (A4-A5), Use (B1-B7), and End of Life (C1- C4). According to the G\&S different lifecycle stages are considered for the analysis both in the case of BIM and Parametric approaches.

\section{Discussion}

According to review papers, BIM and Parametric-based studies usually adopted a different framework to conduct LCA of buildings. This is evident concerning several key aspects. Parametric tools include geometric modelling and scripting functionalities which make it suitable for including several environmental impact categories as seen in Table 1. Most design tools support one or more scripting environments and use a VPL as middleware, which could be bi-directionally linked to BPS tools. Parametric-based LCA usually refers to the early design stages by involving simplified approaches with basic building model. Other studies link Parametric tools with BIM to define a more comprehensive 
approach for conducting LCA. They typically used the BIM model to extract the bill of quantities and Rhino/Grasshopper or Dynamo to generate the LCA calculations as in the case of Hollberg and Ruth (2016). In these cases, Parametric tools are used as a BPS tool since it provides the capacity of scripting and LCA can be performed in the detailed design stages as demonstrated by Shadram and Mukkavaara (2018). Currently, structuring a BIM/Parametric-based framework to perform LCA is quite feasible for all software used, but the interoperability is a challenging task. Several tools are coupled with the modelling software to perform the lifecycle analysis. One of the most important uses of BIM and Parametric tools during the LCA application is to obtain the bill of quantities of materials and components. Table 1 most of the approaches started from the automatic calculation of material quantities that is subsequently exported to a spreadsheet where data are manually managed and organized before developing the environmental impact calculation. LOD is demonstrated to be at the bases of the conception of BIM-based LCA calculations (SoustVerdaguer et al., 2017). Nevertheless, only a few BIMbased studied set the LOD of building model elements. Parametric-based studies, on the other hand, do not refer to the LOD, except for Röck et al. (2018) due to the initial BIM model. Parametric-based approaches are suitable to include several environmental information and then to perform LCA of buildings. This is possible as the VPL software tools provide the capability of creating scripts that reflect mathematical formulations. Usually, BIM is only used to store the geometric information, while Parametric tools can include additional environmental data such as in the cases of Bueno et al. (2018), Hollberg and Ruth (2016), and Lobaccaro et al. (2018). Some BIM-based LCA approaches refer to the early design modelling while others focus on the detailed stage as BIM provides capabilities to model basic and detailed building casestudies. Conversely, most of Parametric- based LCA tools refers to the early design phase since the use of VPL tools is difficult for detailed modelling of buildings. The FU, the database used and the LCA phase considered do not highlight crucial differences between the two approaches (BIM and Parametric approach). That means they are not related to the type of tools or methodologies adopted, but they only refer to the G\&S of the Life Cycle Assessment.

\section{Conclusion}

The paper is based on other papers reviews about BIM and Parametric Based Tools to evaluate the Life Cycle Assessment of buildings. The major findings from previous studies are summarized in Table 1 . The concluding remarks and recommendations for future work in this area are as follows:

- There is a growing interest in integrating LCA into building design decision-making and several tools and methodologies have been developed for BIM and VPL software that adopt a different framework to conduct LCA of buildings.
- Literature reveals how LCA is performed by two different trends, early or detailed design stage; the VPL-based papers analysed focus on the early design stage, while the BIM-based approach refers to both trends depending on the tools used.

- The LCA adoption is tested in different case study, mainly by modelling of complete residential buildings (and few office buildings), mostly developed in Autodesk Revit software to develop the BIM model, while the most widely VPLs are Dynamo and Grasshopper, due to the direct link with Revit and Rhinoceros respectively.

- The definition of the goal and scope $(G \& S)$ of the LCA determines the different lifecycle stages and the selection of environmental impacts to calculate, even if the review reveals how the most calculated environmental impact indicator was GWP both in the case of BIM and VPL; in particular, the inclusion of several environmental impact categories is suitable in VPL due to the scripting functionalities.

A next step of research will consider other criteria such the different way to storage of environmental information in BIM and VPL. To sum up, a long way for building researcher and professionals is still to go in order LCA be more efficient in interoperability and environmentally sustainable.

\section{References}

Abanda, F.H., Oti, A.H. and J.H.M Tah (2017). Integrating BIM and new rules of measurement for embodied energy and CO2assessment. J. Build. Eng. 12, 288-305.

Ajayi, S.O., Oyedele, L.O., Ceranic, B., Gallanagh, M. and K.O Kadiri (2015). Life cycle environmental performance of material specification: a BIMenhanced comparative assessment. Int. J. Sustain. Build. Technol. Urban Dev. 6, 14-24.

Basbagill, J., Flager, F. and M. Lepech (2017). Measuring the Impact of Real-time Life Cycle Performance Feedback on Conceptual Building. $J$. Clean. Prod. 164, 726-735.

Basbagill, J., Flager, F., Lepech, M. and M. Fischer (2013). Application of life-cycle assessment to early stage building design for reduced embodied environmental impacts. Build. Environ. 60, 81-92.

Bueno, C., Pereira, L.M. and M.M. Fabricio (2018). Life cycle assessment and environmental-based choices at the early design stages: an application using building information modelling. Archit. Eng. Des. Manag. 0, 1-15.

Cabeza, L.F., Rincón, L., Vilariño, V., Pérez, G. and A. Castell (2014). Life cycle assessment (LCA) and life cycle energy analysis (LCEA) of buildings and the building sector: A review. Renew. Sustain. Energy Rev. 29.

Cavalliere, C., Dell'Osso, G.R., Pierucci, A. and F. Iannone (2018). Life cycle assessment data structure 
for building information modelling. J. Clean. Prod. 199, 193-204.

Cavalliere, C., Habert, Dell'Osso, G.R. and A. Hollberg (2019). Continuous BIM-based assessment of embodied environmental impacts throughout the design process. J. Clean. Prod. 211, 941-952.

Cheung, F.K.T., Rihan, J., Tah, J., Duce, D. and E. Kurul (2012). Early stage multi-level cost estimation for schematic BIM models. Autom. Constr. 27, 67-77.

Chong, H., Lee, C. and X. Wang (2017). A mixed review of the adoption of Building Information Modelling (BIM) for sustainability. J. Clean. Prod. 142, 4114-4126.

Eleftheriadis, S., Duffour, P. and D. Mumovic (2018). BIM-embedded life cycle carbon assessment of RC buildings using optimised structural design alternatives. Energy Build. 173, 587-600.

Eleftheriadis, S., Mumovic, D. and P. Greening (2017). Life cycle energy efficiency in building structures: A review of current developments and future outlooks based on BIM capabilities. Renew. Sustain. Energy Rev. 67, 811-825.

Georges, L., Haase, M., Houlihan Wiberg, A., Kristjansdottir, T. and B. Risholt (2015). Life cycle emissions analysis of two nZEB concepts. Build. Res. Inf. 43, 82-93.

Hollberg, A. and J. Ruth (2016). LCA in architectural design-a parametric approach. Int. J. Life Cycle Assess. 21, 943-960.

Houlihan Wiberg, A., Georges, L., Dokka, T.H., Haase, M., Time, B., Lien, A.G., Mellegård, S. and M. Maltha (2014). A net zero emission concept analysis of a single-family house. Energy Build. 74, 101-110.

Iddon, C.R. and S.K. Firth (2013). Embodied and operational energy for new-build housing: A case study of construction methods in the UK. Energy Build. 67, 479-488.

International Organisation for Standardisation, (2006). Environmental management-Life Cycle AssessmentPrinciples and Framework (ISO 14040).

Italian National Unification, (2011). Sustainability of construction works - Assessment of environmental performance of buildings - Calculation method (EN 15978).

Jalaei, F. and A. Jrade (2014). An Automated BIM model to conceptually design, analyze, simulate, and assess sustainable building projects. J. Constr. Eng. $1-21$.

Jrade, A. and F. Jalaei (2013). Integrating building information modelling with sustainability to design building projects at the conceptual stage. Build. Simul. 6, 429-444.

Kylili, A., Fokaides, P.A., Vaiciunas, J. and L. Seduikyte (2015). Integration of Building Information Modelling (BIM) and Life Cycle
Assessment (LCA) for sustainable constructions. $J$. Sustain. Archit. Civ. Eng. 4, 28-38.

Lee, S., Tae, S., Roh, S. and T. Kim (2015). Green template for life cycle assessment of buildings based on building information modeling: Focus on embodied environmental impact. Sustain. 7, 16498 16512 .

Lobaccaro, G., Wiberg, A.H., Ceci, G., Manni, M., Lolli, N. and U. Berardi (2018). Parametric design to minimize the embodied GHG emissions in a ZEB. Energy Build. 167, 106-123.

Marzouk, M., Abdelkader, E.M. and K. Al-Gahtani (2017). Building information modeling-based model for calculating direct and indirect emissions in construction projects. J. Clean. Prod. 152, 351-363.

Najjar, M., Figueiredo, K., Palumbo, M. and A. Haddad (2017). Integration of BIM and LCA : Evaluating the environmental impacts of building materials at an early stage of designing a typical office building. $J$. Build. Eng. 14, 115-126.

Negendahl, K. (2015). Building performance simulation in the early design stage: An introduction to integrated dynamic models. Autom. Constr. 54, 3953.

Nizam, R.S., Zhang, C. and L. Tian (2018). A BIM based tool for assessing embodied energy for buildings. Energy Build. 170, 1-14.

Panteli, C., Kylili, A., Stasiuliene, L., Seduikyte, L. and P.A. Fokaides (2018). A framework for building overhang design using Building Information Modeling and Life Cycle Assessment. J. Build. Eng. 20, 248-255.

Peng, C. (2016). Calculation of a building's life cycle carbon emissions based on Ecotect and building information modeling. J. Clean. Prod. 112, 453465.

Röck, M., Hollberg, A., Habert, G. and A. Passer (2018). LCA and BIM: Visualization of environmental potentials in building construction at early design stages. Build. Environ. 140, 153-161.

Schade, J., Olofsson, T. and M. Schreyer (2011). Decision-making in a model-based design process. Constr. Manag. Econ. 29, 371-382.

Shadram, F., Johansson, T.D., Lu, W., Schade, J. and T. Olofsson (2016). An integrated BIM-based framework for minimizing embodied energy during building design. Energy Build. 128, 592-604.

Shadram, F. and J. Mukkavaara (2018). An integrated BIM-based framework for the optimization of the trade-off between embodied and operational energy. Energy Build. 158, 1189-1205.

Shafiq, N., Nurrudin, M.F., Gardezi, S.S.S. and A. Bin Kamaruzzaman (2015). Carbon footprint assessment of a typical low rise office building in Malaysia using building information modelling (BIM). Int. J. Sustain. Build. Technol. Urban Dev. 6, 157-172. 
Shin, Y.S. and K. Cho (2015). BIM application to select appropriate design alternative with consideration of LCA and LCCA. Math. Probl. Eng. 1-15.

Soares, N., Bastos, J., Pereira, L.D., Soares, A., Amaral, A.R., Asadi, E., Rodrigues, E., Lamas, F.B., Monteiro, H., Lopes, M.A.R. and A.R. Gaspar (2017). A review on current advances in the energy and environmental performance of buildings towards a more sustainable built environment. Renew. Sustain. Energy Rev. 77, 845-860.

Soust-Verdaguer, B., Llatas, C. and A. García-Martínez (2017). Critical review of bim-based LCA method to buildings. Energy Build. 136, 110-120.

Wong, J.K.W. and J. Zhou (2015). Enhancing environmental sustainability over building life cycles through green BIM: A review. Autom. Constr. 57, 156-165.

Yang, X., Hu, M., Wu, J. and B. Zhao (2018). Buildinginformation-modeling enabled life cycle assessment, a case study on carbon footprint accounting for a residential building in China. J. Clean. Prod. 183, 729-743. 